\title{
Exploring Expansion and Innovations in Cloud Computing
}

Jitendra Singh, Dyal Singh Evening College, University of Delhi, Delhi, India

\begin{abstract}
Cloud computing is one of the highly sought-after paradigms in information technology. In several cases, it has surpassed earlier predictions of growth, and expanding its services to cover all the key areas. With the growing usage of the cloud, new requirements have also surfaced. To meet user expectations, the cloud services pool has expanded drastically. In order to meet the subscriber's futuristic demands, cloud computing needs to advance further. This work undertakes the study of expansion and innovations that have already happened in the recent past. In addition, perceived cloud evolution in the futuristic cloud has been presented. During the course of exploration, the impact of hardware and software on evolution has been taken into account. Considering the benefits involved, and the current advancement, this work concludes by presenting the innovations that will lead to cloud development.
\end{abstract}

\section{KEYWORDS}

Cloud Containers, DevOps, Emerging Cloud Trend, Hybrid Cloud, Open Cloud, Software Based Infrastructure

\section{INTRODUCTION}

Cloud computing is predicted to be the leading paradigm for the upcoming decade (Singh, 2017). This paradigm is well suited for small and medium enterprises, particularly the one's open to the outside world and need modern, secure, cost effective, and updated resources in terms of harware and software (Singh, Bhisikar, \& Singh, 2013). Availability of infinite hardware resources, pre-build software, anytime anywhere access is some of the key advantages of cloud computing (Singh \& Kumar, 2014a).

In cloud computing, users do not need to pay upfront for resource procurement, instead need to subscribe the required resources by subscribing the cloud services (Mell \& Grance, 2010). User's bill is governed by the size and capabilities of resources subscribed (Buyya, Yeo, Venugopal, Broberg, $\&$ Brandic, 2009). In addition, duration of resource usage is the other factor that determines the bill (Singh \& Kumar, 2014 b; Armbrust, et al., 2009).

The term cloud computing began to gain popularity since 2006, when the companies such as Amazon, Google and Microsoft used this term. Amazon was the first company that commercially launched the cloud in the year 2002 (Mohamed, 2018). The first commercial release of cloud was introduced by Amazon with its Elastic cloud compute (EC2) in the year 2006 (AWS, 2018).

Cloud computing has evolved from several prominent contemporary technologies that includes service-oriented architecture (SOA), web 2.0, virtualization, grid computing, etc. (Singh, 2014). Next generation advancement is governed by the advancement in the cloud enabling technologies, in addition to cloud specific technologies. Virtualization is the other key technology driving the cloud growth. It allows to share the same resources among many users, thereby improves the resource utilization. 
Microsoft is the late entrant in cloud landscape. In 2010, Microsoft entered the cloud landscape with the launch of Windows Azure (Microsoft, 2009). Since then Microsoft cloud computing has expanded their service stack by introducing many new services including Internet of Things (IoT), Big Data, warehousing, etc. Realizing the growth opportunities, new entrants also entered in the cloud business. Now, in every organization irrespective of the type of business, majority of the IT spending is being allocated to cloud. According to IDC, IT speeding of the companies is driven by cloud computing services (Smith \& Shirer, 2018). SaaS will be the driven among the cloud services model (Smith \& Shirer, 2018).

Cloud capabilities can be accessed by subscribing to the virtual machine (VM) offered through the cloud (Singh, 2014). Virtual machines are termed differently according to the cloud. For instance, in amazon web services, VM is termed as instances whereas; in Microsoft azure it is termed as virtual machines. In order to make it cost effective to meet the need of wide variety of users, a number of instances (cloud resources) that are varying in size and capabilities are available and user can subscribe them according to their specific need. Cloud resources can be accessed with the help of variety of devices including Personal computer, Laptop, tablet, Smartphone etc.

Rest of the paper is organized as: Section 2 outlines the cloud services that are dominating in cloud paradigm. Section 3 presents the present trend based on the prevailing technology and the types of services used. Section 3 describes the present trend and Section 4 highlights recent and predicted innovation for cloud paradigm.

\section{CLOUD SERVICES}

In cloud computing, services are offered by cloud service provider. Users can explore and subscribe services according to their requirement. Users may require infrastructure, development environment or the application to serve their routine work. Accordingly, cloud services are categorized into Infrastructure as a Service (IaaS), Platform as a services (PaaS), Software as a services (SaaS) (Mell $\&$ Grance, 2010). Infrastructure requirement such as processor, random access memory (RAM), hard disk, etc., can be accessed from Infrastructure as a Service (IaaS). Variety of service models along with the present trend has been enumerated in the upcoming sub-section.

\section{laas}

Users can install the operating system of their own choice at the top of powerful infrastructure subscribed. Users aspiring for any development environment can subscribe to Platform as a service (Singh \& Kumar, 2013). Whereas, users only need application, they can subscribe to the software as a service (SaaS), thereby applications such as a word processor and Excel can be subscribed to from the cloud. Major cloud providers including Google and Microsoft is already offering Software as a Service. For instance, Google is offering 'Google docs' that can be accessed from the cloud. Microsoft is offering 'office 365 ' to meet the cloud users need related to office application. User seeking specific applications such as ERP, invoice, accounting etc. can subscribe to SaaS and pay based on their usage. Salesforce is the leading SaaS provider primarily dedicated to SaaS.

\section{SaaS}

Application offered through SaaS enjoys several advantages over legacy based. For instance, legacy based application is developed to meet the specific need of a user whereas cloud applications are generic in nature. SaaS based application shortens the development time and avoid any delay that may happen during the development phase. At the same time, offers functionalities that are similar to those of the user base software. This implies that an organization with one personnel is using the full software functionalities that is similar to that of large organizations with 1000 personnel or above. In traditional based system it was not possible due to the cost involved in software development. 
Cloud based application reduces the risk since application developed by established companies is assumed to be robust in functionalities and security relative to the one developed using ad hoc approach. Thereby, all subscribers are exposed to similar resources. Therefore, cloud computing is also termed as democratizing force and offering similar resources to everyone without any discrimination (Singh, 2016).

\section{CLOUD SERVICES PRESENT TREND}

Cloud computing has expanded at a rapid rate. With the growth in cloud computing, new services and applications ranging from word processor to Internet of Things (IoT) have been added in its pool of services (Singh J., 2017). Cloud computing is well suited in scenarios that are exposed to sudden spike in traffic. Such issues can be well-handled by the cloud computing, since, infinite resources can be demanded from the cloud provider. Traffic spike at web portal may need unlimited computing capabilities on the fly that is lacking in legacy system. For instance, traffic at server during last date of filling returns invite massive traffic. To store and analyze such data, we need powerful resources.

\section{Cloud Provider Share}

Cloud paradigm is expanding its services pool by including new services that are compatible to the cloud environment. For instance, Amazon and Microsoft both have added big data services in their pool of services. In other case, growing number of devices in 'Internet of things' is also major source of data generation (Botta, De Donato, Persico, \& Pescape, 2016). As a result, cloud computing has expanded its reach to serve the objective of aforementioned trends. Major cloud providers including Amazon, Microsoft, and Google have already expanded to include these emerging trends.

In cloud domain, Microsoft, Amazon, IBM, Google are the leading cloud service provider (Singh, 2017). Majority of the cloud share is held by the aforementioned companies. In addition, new cloud providers are also emerging and offering specialized services in IaaS, PaaS, SaaS domain. Revenue share of cloud providers in IaaS is illustrated in Figure 1.

According to the Figure 1, Amazon holds majority of the share in IaaS, this is followed by Microsoft azure; third position is held by Google Cloud, and this is followed with IBM. Beyond the major cloud providers, share of new cloud providers is also substantial, and amounts to around 36 percent. Increasingly important to note that the share of Google is not at par with the other cloud provider, despite it holds high specialization in cloud domain.

Surprisingly, in the fourth quarter of 2017, IBM has topped in revenue generation and pushed Microsoft and AWS to third and fourth position respectively (Evans, 2018). Revenue generation of major cloud provider has been illustrated in Figure 2. Numbers are alarming and it appear that market share of major cloud provider may shift from one to another.

Encouraged with growing user's subscription, Cloud services are expanding by adding new services at regular interval. As a result, cloud providers services offering have grown manifold. Services in database, storage, data warehousing etc. are included in the service pool of cloud provider and now it is offered by majority of the cloud providers. Although issues in big data were raised, however, there is no evidence in real life has been witnessed (Hashem, et al., 2015). Leading cloud providers and their offering nomenclature has been presented in Table 1.

\section{EMERGING TREND IN CLOUD COMPUTING}

Cloud computing is believed to be the leading paradigm that will drive the future. After envisioned, it has transformed itself to a great extent in order to meet the requirement of large user base. To proliferate further, it needs to expand itself by introducing innovation. Upcoming section discusses the trend and advances that will lead the cloud paradigm. 
Figure 1. laaS platform adoption-percentage of application deployed (Mcafee, 2017)

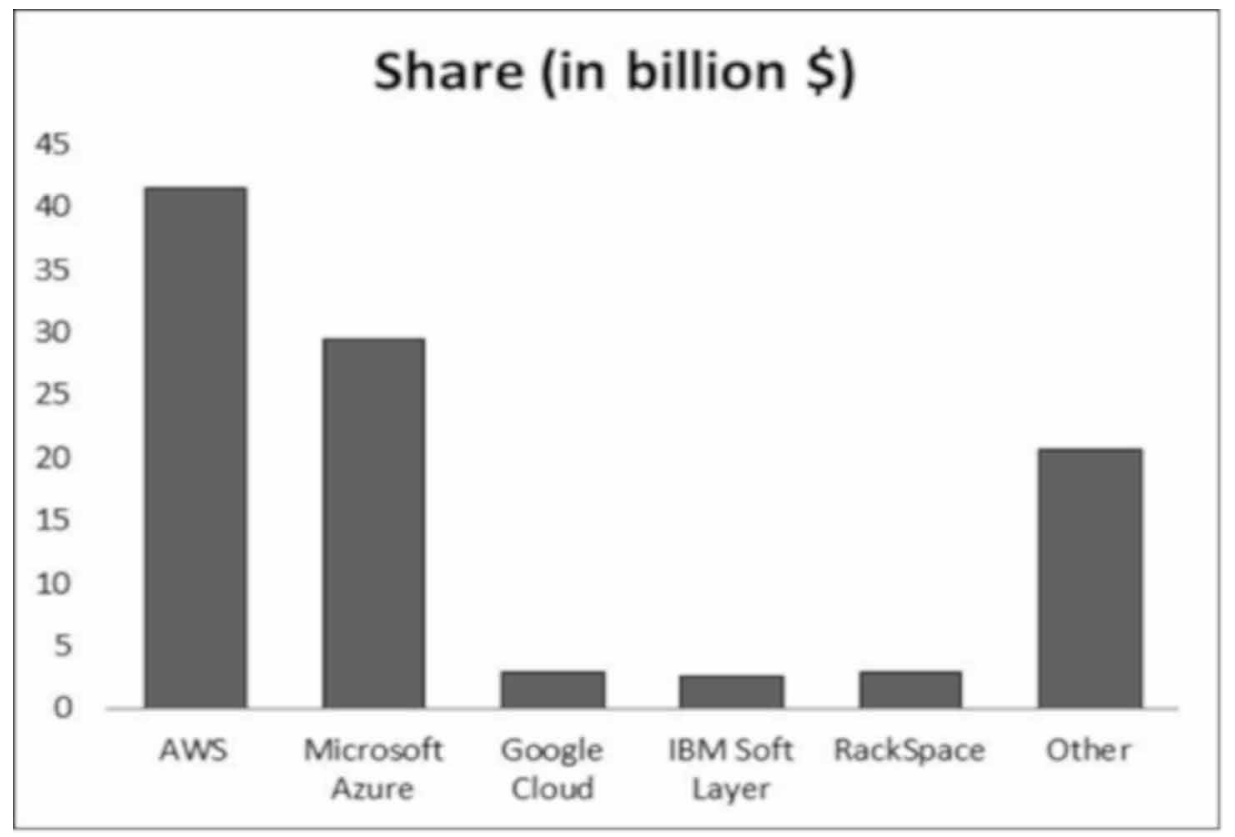

Figure 2. Cloud provider Revenue Q4 2017 (Evans, 2018)

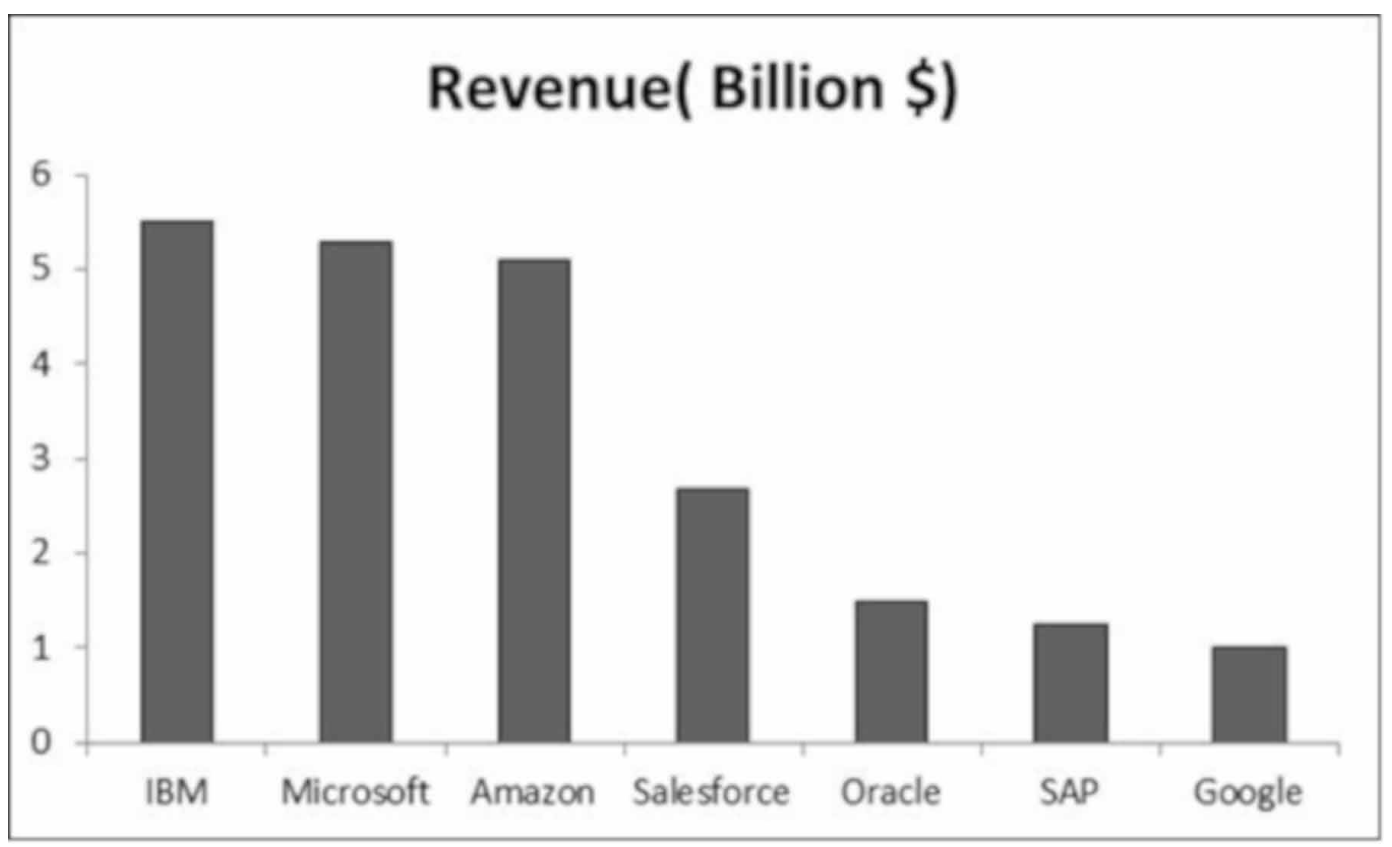




\begin{tabular}{|c|c|c|}
\hline Services & Microsoft Azure & Amazon Web Services \\
\hline \multirow{3}{*}{$\begin{array}{l}\text { Relational } \\
\text { DBMS }\end{array}$} & Data Factory & Aurora \\
\hline & Azure SQL Database & Elastic Cache \\
\hline & Redis Cache & Amazon RDS \\
\hline \multirow{4}{*}{ Storage } & $\begin{array}{l}\text { StorSimple, Data Lake } \\
\text { Store }\end{array}$ & Amazon 53 \\
\hline & BloB Store & AWS SnowMobile \\
\hline & Queue Storage & AWS Snowball \\
\hline & Azure Cosmos DB & \\
\hline $\begin{array}{l}\text { Internet of } \\
\text { Things(IOT) }\end{array}$ & $\begin{array}{l}\text { loT Hub, Azure Sphere, } \\
\text { Stream Analytics, IoT } \\
\text { Edge }\end{array}$ & $\begin{array}{c}\text { IOT Core, AWS IOT Device } \\
\text { Managemen, AWS IoT } \\
\text { Device Defender }\end{array}$ \\
\hline Data Analytics & $\begin{array}{l}\text { SQL Data Warehouse, } \\
\text { Azure Databricks, Queue } \\
\text { Storage,Azure Cosmos } \\
\text { DB }\end{array}$ & $\begin{array}{l}\text { Amazon Athena, Amazon } \\
\text { Kinesis, Amazon RedShift }\end{array}$ \\
\hline Compute & $\begin{array}{l}\text { Virtual Machines, Azure } \\
\text { Batch Al, Service Fabric }\end{array}$ & $\begin{array}{l}\text { EC2,Amazon Elastic } \\
\text { Search Service, AWS } \\
\text { Lambda }\end{array}$ \\
\hline Security & $\begin{array}{l}\text { Security Center, Key } \\
\text { Vault, Azure Ddos } \\
\text { Protection }\end{array}$ & $\begin{array}{l}\text { amazon Shield, Amazon } \\
\text { WAF, Amazon } \\
\text { Insspecctor }\end{array}$ \\
\hline
\end{tabular}

\section{Emergence of Hybrid Cloud}

Once user is maintained on premises private cloud at the same time subscribed to the public cloud then this arrangement is termed as hybrid cloud. In hybrid cloud model, data transfer occurs seamlessly (Rittinghouse \& Ransome, 2016). It is believed that this model facilitates harnessing the cloud based capabilities along with on premises model. Subscriber maintaining the sensitive data would continue to use the on premises model (Singh \& Kumar, 2011). In order to harness the cloud services, they may use public cloud for less sensitive data and public cloud where the resource demand surges immediately.

According to large number of predictions, hybrid cloud model has great potential to grow in upcoming years. According to Sandler Research's, hybrid cloud will witness tremendous growth. Hybrid model may witness the CAGR of around 28 percent (Sandlech Research, 2015). A large number of leading organizations across the world have already embraced the hybrid model including Amazon, Microsoft in order to harness the benefits of cloud. With the passage of time, hybrid model in addition to cloud-based security may gain momentum to cater the need of SMEs (Singh \& Kumar, 2011).

VMware in its publication anticipated constinuous growth in hybrid cloud and introduced measure to assess pricing in private and public cloud, in order to ensure that appropriate type of cloud is selected (VmWare, 2017). To justify their claim of growth, e-commerce, archiving, outsource IT enterprises, package application etc. are cited as the key reasons that will drive the growth in hybrid cloud. All the aforementioned domain will substantially reduce the IT expenditure, cause resource upgradation, and multiple sites maintenance to meet the regulatory compliance in the event of calamity or natural disaster (Singh \& Kumar, 2014b; Singh \& Kumar, 2013). Realizing high growth potential, existing vendors are proliferating their services. In addition, new are emerging (Chou, 2015).

Hybrid cloud is well supported by Microsoft. User can maintain the private cloud with the help of Windows 2012 R2 operating system or its higher version. At the same time, they can harness the benefit of public cloud by subscribing to the Microsoft public cloud i.e. windows Azure. Amazon is 
also supporting the hybrid cloud. In Amazon's hybrid model, user can use the eucalyptus cloud on their premises and Amazon web services (AWS) as public cloud.

\section{Advances in Regulatory Standard}

Due care is being exercised by the cloud providers in order to safeguards the subscriber data, maintain privacy and security (Chang, Kuo, \& Ramachandran, 2016). However, countries remained equally concern on the privacy and security of their citizen's data. Accordingly, regulations are passed from time to time in order to meet the contemporary need. However, in case of cloud paradigm, many regulations are not framed. Instead, most of the regulations applicable in legacy-based system are only applied (Singh \& Kumar, 2014a). Majority of us still treat cloud paradigm as client serverbased model only. Indeed, there is substantial variation from legacy-based system to that of cloud computing. Correspondingly, additional regulations need to be framed since subscribers do not only belong to one specific country instead from a large number of countries (Ali, Khan, \& Vasilakos, 2015; Singh \& Raghuvanshi, 2017).

Similarly, in a country where the subscriber resides also need to modified the regulation to categorize the data into sensitive and non-classified, etc., in order to allow storage away from the country based on the category in which data falls (Bolgert, Kalyanaraman, Forlenza, \& Cohen, 2015; Ferris, 2015).

\section{Devops}

DevOps is combination of cultural philosophies, practices, and its tools that increases an organization's ability to deliver applications and services at a high velocity. Evolving and improving products at a faster pace than organization using legacy software development and infrastructure processes. This helps the business to serve the customer better instead of concentrating on Information Technology.

Under DevOps model, teams are no longer working in silos instead member of one team can contribute in other teams. This leads to strong understanding of a project, at the same time eliminates the delay that may be caused due to team's coordination. All the team members are adopting process in order to automate it. As a result, overall speed of deployment enhances. Automation tools in assistance, engineers can accomplish the task independently. Speed, rapid delivery, reliability, scalability, improved collaborations etc. are some of the significant features of DevOps. It is the philosophy that begins with making the platform visible to everyone. In short, it deals with advance development tool to meet the demand of modern-day business scenario.

\section{Adoption of Container Technologies}

Containers is an emerging trend in cloud computing and primarily a virtualization enabler that is similar to that of virtual machine in legacy-based system. However, there is large difference between the virtualization offered by virtual machine and the one in containers. Virtual machine-based virtualization is working at operating system level and shares the hardware resources, whereas, container virtualization is acting at application layer and do not share the resources. Virtualization used by containers is a light version relative to the one used by virtual machine. Containers separate users in their virtualized compartment. Thereby, offers high degree of security and isolation among users.

Container based virtualization is well suited in cloud paradigm that is driven by same resource availability among all the subscribers. In addition, its docking feature enables it to shift the container from one server to another on the fly. Docking feature is highly sought in paradigm such as cloud computing, where the user workload can be shifted to nearby data center in the event of failure. Docking will ensure higher uptime in cloud paradigm, as a result higher satisfaction among subscribers. Leading cloud providers including Microsoft, Amazon, and IBM etc. have already embraced container technology to exploit their resources and to minimize the operational expenses. Container technologies reduces the operating cost without compromising the security and user's experience. 


\section{Multi Cloud Subscription}

Multi cloud subscription proved to be worthy in several cases. For instance, in case of commonwealth of Australia (Schlagwein, Thorogood, \& Willcocks, 2014). However, in present day scenario, majority of the cloud providers are using their own specific protocol and Application programming interface (API) for data storage and data rendering. Consequently, data stored in one cloud is not portable to another cloud (Bracci, Corradi, \& Foschini, 2014). This is one of the major concerns among cloud subscribers that what happens to their data once they migrate to another cloud provider in the event of non-satisfaction that may arise due to poor services or the method of billing.

Several organizations are working in order to developing the standard that should be inter-operable (CDMI; Crosby, et al., 2010). Once, such standard is evolved and adopted by the leading as well as emerging cloud providers then it will be appreciated by all the stakeholders. Users can subscribe to any cloud provider without any apprehension. In addition, user may be using the infrastructure of one cloud provider, development environment of another cloud provider and the applications of third cloud provider (Metsch, Edmonds, \& others, 2011). This can only put into practice, if all the cloud adopts the same standard and API's (Crosby, et al., 2010). Consequently, user will able to subscribe to multi cloud (OMG). Presently, subscription to multi cloud is cumbersome and can be achieved by subscribing to the providers such as Right Scale that facilitates in connecting multiple vendors. Currently, it can be accomplished with the help of cloud broker. However, using cloud broker need training in order to carry out operation on multi cloud.

\section{Continuous Delivery}

Cloud outage is other major issue that exist in cloud computing. Every year cloud subscribers are meeting with huge losses due to the cloud outage (Singh, 2014). Since, it is profoundly new paradigm, new challenges are emerging frequently. As a result, subscribers are experiencing poor experience. Even worst, users working as third party to host the services of consumers invite severe criticism or even loss of reputation and revenue. According to International Working Group on Cloud Computing Resiliency (IWGCR), huge losses are incurred annually due to non-availability of services. All the cloud providers irrespective of revenue share they are holding, encountering cloud outage (http:// iwgcr.org). According to the IWGCR, only major cloud outages are being reported and those of short duration passes unnoticed.

\section{Open Cloud}

Cloud computing adheres to two types of API for its functioning the first type is termed as Proprietary and the second one as open. Proprietary API's are vendor specific whereas open API's are designed on global standards to ensure the homogeneity among cloud vendors. In cloud paradigm, lack of interoperability is considered as the major hindrance in cloud growth. To address this issue three bodies have emerged and managing the standards in order to ensure that the application developed for one cloud can easily migrated to another cloud providers. Standard bodies contributing with respect to interoperability are presented herein (CDMI; OMG; SNIA):

- $\quad$ Open Cloud Computing Interface (OCCI)

- Cloud Data Management Interface (CDMI)

- Open Virtualization Format (OVF)

To address the interoperability issue, Open cloud computing interface (OCCI) has outlined the guidelines that are based on REST (Metsch, Edmonds, \& others, 2011). Cloud complying with the OCCI guidelines will be inter-operable since they won't be using their own API instead adopting the signature and identification as outlined by the OCCI and termed as open cloud (Rochwerger et al., 2009). 
In cloud computing, virtualization plays a key role, however, lacks common standard at cloud service provider end. 'Open virtualization format (OVF)' is proposed as standard for virtualization across all the cloud provider (Shah, Elzur, \& Thaler, 2013). However, it is not complied by many cloud providers. Even majority of them have not revealed information pertaining to the adoption of OVF. Similarly, other standards covering cloud storage and other services have emerged in order to facilitate the cloud interoperability.

Cloud Data Management Interface (CDMI) deals with the standard related to the storage. It specifies the way information is to be create, stored, retrieved, or delete the data element during storage (Wu, Ping, Ge, Wang, \& Fu, 2010). Developers adopting CDMI can easily migrate their app from one cloud to another by following the CDMI.

Recently, IT pioneer IBM has introduced the open cloud naming IBM open cloud, that is motivated with the 'Open Stack's' open cloud. In IT industry, proprietary paradigm had never dominated; instead it proliferated once the users are offered choice to adopt anything. Accordingly, open cloud will grow further. Cloud provider's using the proprietary APIs will gradually shift to open standards in order to compete other vendors at the same time meeting the global standards and compliance. Open cloud will also facilitate the portability of apps from one cloud to another. In other words, web powered apps dream will come true. IBM open cloud has adopted the OVF architecture. Open standards are the key in the direction of cloud interoperability.

\section{Software Based Infrastructure}

Cloud computing is about the economy of resources by way of optimizing the resource utilization. Optimum resource utilization will result in lowering usage cost for cloud provider; consequently, profit of cloud service provider will grow. In software-based infrastructures, resources will be allocated with the help of virtualization. Users will not be able to distinguish between the software based and hardware based resourced ( $\mathrm{Li} \&$ Chen, 2015). Indeed, software based infrastructure offers the physical infrastructure by sharing it among the users. Software resources are appreciated due to the greater flexibility to the subscribers to meet their wider need (Cui, Yu, \& Yan, 2016). Trend of softwarebased resources will grow further to include wider customer base. In addition to the software-based infrastructure, software based networking is also gaining momentum (Li \& Chen, 2015; Yan \& Yu, 2015). Currently, VMware is offering its virtualization-based software for network utilization. In upcoming years trend of virtualization will grow further. To improve resource utilization, (Moshref, Yu, Govindan, \& Vahdat, 2015) have suggested the dynamic resource allocation, where the load will be shifted to poorly dense data center from the one densely allocated.

\section{Performance Improvement}

Performance is a key issue in cloud computing. In present day computing environment, users are aiming for rich performance experience similar to that of their own network. Since, cloud is Internet based access of resources, latency and connectivity are the key issues governing users experience. Internet bandwidth has surged manifold in last few years. For instance, in 2001, only limited speed was available to us. Now, it has grown manifold. Enhancement in network bandwidth could be possible due to spread of fiber optical cable in developed and developing countries.

In addition, to meet performance demand, cloud specific devices are being developed. For instance, recently Google has developed cloud computing dedicated devices with the name Chrome Notebook. It is gaining positive response from the user's community. To offer all round connectivity, several layers of Internet has been provisioned. In the event if one connection fails it will switch over to another one available in the pool. Beyond, this devices identification number will assist in countering the cyberattacks. Existing mechanical based storage device is replaced with fast and reliable SSD based storage. To boost the speed at its data center, Google has already deployed the SSD based storage. It uses four replication layers to ensure the data security in the event of failure. Thereby, such devices will bring significant improvement in performance. 


\section{Country Specific Data Center}

Cloud data center location is profoundly sensitive for both the cloud provider as well as subscriber. Data center location is selected based on several factors including security, availability of electricity and skill set, government regulations etc. Countries already established themselves as peaceful and rich in resources in terms of electricity and human skill are opted first. Since, availability is the major concern in cloud paradigm therefore, data center needs uninterrupted power supply. In addition, security at the location selected is another major concern. If country with presence of terrorist activity is chosen in that case subscriber from other country won't opt for such cloud data center.

In majority of cases, cloud data is stored at data center that are located at developed countries. Data center in emerging countries are lacking. For instance, the leading cloud provider Amazon is serving the subscribers around the world with its data center located in USA or in European countries (Singh \& Kumar, 2014a). Same is true with another leading cloud provider Microsoft. This leads to generation of concern related to data security and privacy among the countries not enjoying close ties with USA and Europe.

Storing the data outside of subscriber's country is another major challenge related to security and compliance that is hindering the cloud growth (Ab Rahman \& Choo, 2015; Duncan \& Whittington, 2017; Duncan \& Whittington, 2016). Emerging trend will be locating the data at developed countries. In some cases region will be figured out where the several friendly countries are located. Region is to be selected that is meeting the majority of the aforementioned factors of data center location. For instance, India and Nepal are the friendly country and locating the data center at India will not be objected by Nepal since later enjoys close tie-up with the former. At the same time, data center at India location will serve the major market for cloud provider.

\section{Full Virtualization of the Resources}

Virtualization is the key enabler for the cloud computing, since it facilitates the resource sharing among many users. Although it is widely used in cloud paradigm, we perceive the trend will grow further for higher performance and sound security system. Virtualization will also be needed to meet the dynamic and variant demand of the subscribers. Presently virtualization is already used at various level one at hardware level when the virtualization software interface directly to the hardware and at the top of it resides several operating system. This layer is termed as Virtual machine manager (VMM). Hyper-V, Xen, KVM, Esxi etc. are leading VMM that are facilitating the hardware level virtualization (Singh, 2014).

Virtualization layer can also reside at the top of software termed as software-based virtualization. But software-based virtualization meets with performance bottleneck. With the passage of time, virtualization will able to grow further, at the same time it will be lighter thereby it can be migrated from one data center to another with minimum loss. In addition, virtualization is used in data center, networking to name a few.

VMware is the pioneer in virtualization technology and hold the substantial market share. Expert perceived that soon virtualization-based solution will exist to overcome hardware bottleneck. In virtualization, VMware is followed by Citrix. In virtualization, logical partition of same resources is carried out that need strong protection among the user's environment. VMware's vShield is a solution that safeguards the virtualized environment.

\section{Noops and Serverless Architecture}

Once user decides migration from legacy to the cloud services, one need to understand a lot related to cloud technical jargon involved in order to exploit the functionality of cloud paradigm. In addition, a lot of parameters need to be supplied in order to generate and switch-on the instance and assigning load to it. All such actions need skilled professional in order to leverage the cloud paradigm. Although, wizards facilitate the user during provision of virtual machine and assigning the load, yet it does not minimize 
the parameter required that are needed to propel the cloud growth due to ease of usage. Precisely to maintain IT resources, team of reasonable size is needed that result in excessive expenditure.

Noops is the concept that signifies extensive use of automation by abstracting the underlying layers. The key reason is to offer ease to an extent that it can be operated and managed without the need of dedicated team. According to the Forrester "the goal of completely automating the deployment, monitoring and management of applications and the infrastructure on which they run." According to Forrester Senior Analyst Glenn O'Donnell, who co-authored the report “Augment DevOps with NoOps." Organizations will be termed as noops organization that will not have any person to manage the operations. Several cloud providers for instance Heruku and Appfog among others claiming to be Noops based. Debate on the need of DevOps is also emerging. However, we perceive that Noops will serve the generic requirement whereas Devops will begin where the Noops unable to meet the requirement.

Popularity of cloud will grow further due to the application of abstraction at bottom layer that is responsible for implementation of underlying technology. Therefore, the term Noops.

Other perceived implementation is lying with server-less architecture. In this architecture, resources will be allocated based on the services sought and will offer best fit considering application into account. To this end, machine learning based techniques are being developed. This will also lead to optimum utilization of resources at the same time result in dropping subscriber's bill substantially.

In forthcoming years, more automation tool to the tune of Noops will emerge that would require no operation from the user end. Activities such as machine generation to load assignment can be carried out in minimum human intervention.

\section{Popularization of Nonvolatile RAM (NVRAM)}

Memory is the essential part of a computer system. Prior to execution of data, it needs to be brought into memory. Post execution, processed data is placed back into memory. During fetch and store operation, processor need to wait for long time since speed of RAM is increasingly low relative to the one offered by processor (McKnight, Zuo, \& Maheshwari, 2015). Thus, a number of processing cycles result in no computation. Although, cache has been introduced to lighten the loss that may occur due to high speed mismatch, yet loss occurs. As a result, processor is not able to perform to its fullest capacity. In addition, in the event of power failure, data stored in RAM will be lost. Since, RAM inherits the property of volatility that implies that data is retained only for the duration the power is on. In the event of failure, data will be lost. However, when such things happen, it leads to loss of data that is undesired. To overcome this limitation, a new technique termed as NVRAM has evolved (Huang, Schwan, \& Qureshi, 2014). As the name Nonvolatile itself suggests that the RAM is nonvolatile in nature. Therefore, no data loss will occur in the event of failure of a disk.

\section{CONCLUSION}

Cloud paradigm has established itself as highly sought paradigm in IT landscape. In order to meet the subscribers need, it is expanding its services rapidly. To this end, it needs to adopt new concept and principles in order to accommodate present and futuristic demand. At the same time, it need to address the wider concern of subscriber related to security, reliability, interoperability, performance etc. This will only be possible if cloud providers adopts new technology that is efficient, reliable at the same time cost effective. Once all the aforementioned objectives are fulfilled then only a true and widely accepted cloud paradigm dream will be true. 


\section{REFERENCES}

Ab Rahman, N. H., \& Choo, K.-K. R. (2015). A survey of information security incident handling in the cloud. Computers \& security, 49, 45-69.

Ali, M., Khan, S. U., \& Vasilakos, A. V. (2015). Security in cloud computing: Opportunities and challenges. Information Sciences, 305, 357-383. doi:10.1016/j.ins.2015.01.025

AWS. (2018). About AWS. Retrieved from https://aws.amazon.com/about-aws/

Bolgert, A. L., Kalyanaraman, R., Forlenza, R. M., \& Cohen, R. J. (2015). U.S. Patent No. 9,110,976. Washington, DC: U.S. Patent and Trademark Office.

Botta, A., De Donato, W., Persico, V., \& Pescape, A. (2016). Integration of cloud computing and internet of things: A survey. Future Generation Computer Systems, 56, 684-700. doi:10.1016/j.future.2015.09.021

Botta, A., De Donato, W., Persico, V., \& Pescapé, A. (2016). Integration of cloud computing and internet of things: A survey. Future Generation Computer Systems, 56, 684-700. doi:10.1016/j.future.2015.09.021

Bracci, F., Corradi, A., \& Foschini, L. (2014). Cloud Standards: Security and Interoperability Issues. In Communication Infrastructures for Cloud Computing (pp. 465-495). Hershey, PA: IGI Global.

Buyya, R., Yeo, C. S., Venugopal, S., Broberg, J., \& Brandic, I. (2009). Cloud computing and emerging IT platforms: Vision, hype, and reality for delivering computing as the 5th utility. Future Generation Computer Systems, 25(6), 599-616. doi:10.1016/j.future.2008.12.001

CDMI. (n.d.). CDMI Cloud Storage Standard. (SNIA) Retrieved from https://www.snia.org/cloud/cdmi

Chang, V., Kuo, Y.-H., \& Ramachandran, M. (2016). Cloud computing adoption framework: A security framework for business clouds. Future Generation Computer Systems, 57, 24-41. doi:10.1016/j.future.2015.09.031

Chou, D. C. (2015). Cloud computing: A value creation model. Computer Standards \& Interfaces, 38, $72-77$.

Crosby, S., Doyle, R., Gering, M., Gionfriddo, M., Grarup, S., Hand, S., et al. (2010). Open virtualization format specification.

Crosby, S., Doyle, R., Gering, M., Gionfriddo, M., Grarup, S., Hand, S., Hapner, M., Hiltgen, D., et al. (2010). Open virtualization format specification.

CSA. (2013). The Notorious Nine:Cloud Computing Top Threats in 2013. Retrieved from https://downloads. cloudsecurityalliance.org/initiatives/top_threats/The_Notorious_Nine_Cloud_Computing_Top_Threats_in_2013. pdf

Cui, L., Yu, F. R., \& Yan, Q. (2016). When big data meets software-defined networking: SDN for big data and big data for SDN. IEEE Network, 30(1), 58-65. doi:10.1109/MNET.2016.7389832

Cui, L., Yu, F. R., \& Yan, Q. (2016). When big data meets software-defined networking: Sdn for big data and big data for sdn. IEEE Network, 30(1), 58-65. doi:10.1109/MNET.2016.7389832

Duncan, R. A., \& Whittington, M. (2016). Enhancing cloud security and privacy: the power and the weakness of the audit trail. In Cloud Computing 2016.

Duncan, R. A., \& Whittington, M. (2017). Creating an Immutable Database for Secure Cloud Audit Trail and System Logging. In Eighth International Conference on Cloud Computing, GRIDs, and Virtualization, February 19-23, Athens, Greece.

Evans, B. (2018, Feb). Why Microsoft Is Ruling The Cloud, IBM Is Matching Amazon, And Google Is $\$ 15$ Billion Behind. Forbes. Retrieved from https://www.forbes.com/sites/bobevans1/2018/02/05/why-microsoft-isruling-the-cloud-ibm-is-matching-amazon-and-google-is-15-billion-behind/\#580a9411dc1a

Ferris, J. M. (2015). U.S. Patent No. 9,052,939. Washington, DC: U.S. Patent and Trademark Office.

Fox, A., Griffith, R., Joseph, A., Katz, R., Konwinski, A., Lee, G., \& Stoica, I. (2009). Above the clouds: A berkeley view of cloud computing. Dept. Electrical Eng. and Comput. Sciences. Berkeley: University of California. 
Hashem, I. A., Yaqoob, I., Anuar, N. B., Mokhtar, S., Gani, A., \& Khan, S. U. (2015). The rise of "big data" on cloud computing: Review and open research issues. Information Systems, 47, 98-115. doi:10.1016/j. is.2014.07.006

Hashem, I. A. T., Yaqoob, I., Anuar, N. B., Mokhtar, S., Gani, A., \& Khan, S. U. (2015). The rise of "big data" on cloud computing: Review and open research issues. Information Systems, 47, 98-115. doi:10.1016/j. is.2014.07.006

Huang, J., Schwan, K., \& Qureshi, M. K. (2014). NVRAM-aware logging in transaction systems. Proceedings of the VLDB Endowment International Conference on Very Large Data Bases, 8(4), 389-400. doi:10.14778/2735496.2735502

Huang, J., Schwan, K., \& Qureshi, M. K. (2014). Nvram-aware logging in transaction systems. Proceedings of the VLDB Endowment International Conference on Very Large Data Bases, 8(4), 389-400. doi: $10.14778 / 2735496.2735502$

ISO. (2014). ISO/IEC 17789:2014: Information technology -- Cloud computing -- Reference architecture. Retrieved from https://www.iso.org/standard/60545.html

Kalaiprasath, R., Elankavi, R., Udayakumar, D. R., \& others. (2017). Cloud. Security and Compliance-A Semantic Approach in End to End Security. International Journal Of Mechanical Engineering And Technology, 8.

Khan, M. A. (2016). A survey of security issues for cloud computing. Journal of Network and Computer Applications, 71, 11-29. doi:10.1016/j.jnca.2016.05.010

Li, Y., \& Chen, M. (2015). Software-defined network function virtualization: A survey. IEEE Access, 3 , 2542-2553. doi:10.1109/ACCESS.2015.2499271

Li, Y., \& Chen, M. (2015). Software-defined network function virtualization: A survey. IEEE Access, 3, 2542-2553. doi:10.1109/ACCESS.2015.2499271

Martinez, F. R., \& Pulier, E. (2015). U.S. Patent No. 9,069,599. Washington, DC: U.S. Patent and Trademark Office.

Mcafee. (2017). Custom Applications and IaaS Trends. Retrieved from http://info.skyhighnetworks.com/rs/274AUP-214/images/wp-csa-survey-custom-apps-iaas-survey-report.pdf

McKnight, T. P., Zuo, X., \& Maheshwari, U. (2015). U.S. Patent No. 8,949,502. Washington, DC: U.S. Patent and Trademark Office.

McKnight, T. P., Zuo, X., \& Maheshwari, U. (2015). U.S. Patent No. 8,949,502. Washington, DC: U.S. Patent and Trademark Office.

Mell, P., \& Grance, T. (2010). The NIST definition of cloud computing. Communications of the ACM, 53, 50.

Mell, P., \& Grance, T. (2010). The NIST definition of cloud computing. Communications of the ACM, 53(6), 50.

Mell, P. \& Grance, T. (2011). The NIST definition of cloud computing.

Metsch, T., Edmonds, A., \& others. (2011). Open cloud computing interface-RESTful HTTP rendering. Open Grid Forum.

Metsch, T., \& Edmonds, A. (2011). Open cloud computing interface-restful http rendering. In Open Grid ForumOCCI Working group technical report.

Microsoft. (2009, Nov 17). Microsoft Cloud Services Vision Becomes Reality With Launch of Windows Azure Platform. Retrieved from https://news.microsoft.com/2009/11/17/microsoft-cloud-services-vision-becomesreality-with-launch-of-windows-azure-platform/

Mohamed, A. (2018). A history of cloud computing. Computer Weekly. Retrieved from https://www. computerweekly.com/feature/A-history-of-cloud-computing

Moshref, M., Yu, M., Govindan, R., \& Vahdat, A. (2015). DREAM: Dynamic resource allocation for softwaredefined measurement. Computer Communication Review, 44(4), 419-430. doi:10.1145/2740070.2626291 
Moshref, M., Yu, M., Govindan, R., \& Vahdat, A. (2015). Dream: Dynamic resource allocation for softwaredefined measurement. Computer Communication Review, 44(4), 419-430. doi:10.1145/2740070.2626291

OMG. (n.d.). Object Management Group. Retrieved from https://www.omg.org/memberservices/index.htm

Rittinghouse, J. W., \& Ransome, J. F. (2016). Cloud computing: implementation, management, and security. CRC press.

Rochwerger, B., Breitgand, D., Levy, E., Galis, A., Nagin, K., Llorente, I. M., \& Galan, F. et al. (2009). The reservoir model and architecture for open federated cloud computing. IBM Journal of Research and Development, 53(4), 4-1. doi:10.1147/JRD.2009.5429058

Rochwerger, B., Breitgand, D., Levy, E., Galis, A., Nagin, K., Llorente, I. M., \& Galan, F. et al. (2009). The reservoir model and architecture for open federated cloud computing. IBM Journal of Research and Development, 53(4), 4-1. doi:10.1147/JRD.2009.5429058

Schlagwein, D., Thorogood, A., \& Willcocks, L. P. (2014). How Commonwealth Bank of Australia gained benefits using a standards-based, multi-provider cloud model. MIS Quarterly Executive, 13.

Shah, H., Elzur, U., \& Thaler, P. A. (2013). U.S. Patent No. 8,417,800. Washington, DC: U.S. Patent and Trademark Office.

Singh, H. P., Bhisikar, A., \& Singh, J. (2013). Innovative ICT Through Cloud Computing. IUP Journal of Computer Sciences, 7.

Singh, J. (2014). Cloud Computing for Beginner to researcher. CreateSpace.

Singh, J. (2014). Cyber-attacks in cloud computing: A case study. International Journal of Electronics and Information Engineering, 1, 78-87.

Singh, J. (2017). Study on Challenges, Opportunities and Predictions in Cloud Computing. International Journal of Modern Education and Computer Science, 9(3), 17-27. doi:10.5815/ijmecs.2017.03.03

Singh, J., \& Kumar, V. (2011). Assessment of security risks in merging the private and public cloud. Asia Pacific Business Review, 7, 180-190.

Singh, J., \& Kumar, V. (2013). Compliance and Regulatory Standards for Cloud Computing. In Interdisciplinary Perspectives on Business Convergence, Computing, and Legality (pp. 54-64). Hershey, PA: IGI Global. doi:10.4018/978-1-4666-4209-6.ch006

Singh, J., \& Kumar, V. (2013). Implementation of User-End Broker Policy to Improve the Reliability of Cloud Services. International Journal of Cloud Applications and Computing, 3(4), 13-27. doi:10.4018/ijcac.2013100102

Singh, J., \& Kumar, V. (2014a). Virtual Appliances-Based Framework for Regulatory Compliances in Cloud Data Centers. IUP Journal of Information Technology, 10, 30.

Singh, J., \& Kumar, V. (2014 b). Multi-disciplinary research issues in cloud computing. Journal of Information Technology Research, 7(3), 32-53. doi:10.4018/jitr.2014070103

Singh, J., Powles, J., Pasquier, T., \& Bacon, J. (2015). Seeing through the clouds: Management, control and compliance for cloud computing. Cloud Computing, 1-12.

Singh, J., \& Raghuvanshi, K. (2017). Study on the Development of Cloud Security. Special Issue of Engineering and Scientific International Journal.

singh, j. (2016). Democratizing IT: A Cloud Computing Framework. International journal of education and management engineering, $6,1-13$.

Smith, E., \& Shirer, M. (2018, Jan. 18). Worldwide Public Cloud Services Spending Forecast to Reach \$160 Billion This Year, According to IDC. IDC. Retrieved from https://www.idc.com/getdoc.jsp?containerId=prUS43511618

SNIA. (n.d.). Open Grid Form. Retrieved from https://www.snia.org/about/alliances/ogf

VmWare. (2017). Hybrid Cloud Assessment. VMware. 
Wang, B., Zheng, Y., Lou, W., \& Hou, Y. T. (2015). DDoS attack protection in the era of cloud computing and software-defined networking. Computer Networks, 81, 308-319. doi:10.1016/j.comnet.2015.02.026

Wang, B., Zheng, Y., Lou, W., \& Hou, Y. T. (2015). Ddos attack protection in the era of cloud computing and software-defined networking. Computer Networks, 81, 308-319. doi:10.1016/j.comnet.2015.02.026

Wang, L., \& Ranjan, R. (2015). Processing distributed internet of things data in clouds. IEEE Cloud Computing, 2(1), 76-80. doi:10.1109/MCC.2015.14

Wayne W. Armour, N. B. (2013). NIST Cloud Computing Security Reference Architecture. NIST.

Wei, L., Zhu, H., Cao, Z., Dong, X., Jia, W., Chen, Y., \& Vasilakos, A. V. (2014). Security and privacy for storage and computation in cloud computing. Information Sciences, 258, 371-386. doi:10.1016/j.ins.2013.04.028

Wu, J., Ping, L., Ge, X., Wang, Y., \& Fu, J. (2010). Cloud storage as the infrastructure of cloud computing. In 2010 International Conference on Intelligent Computing and Cognitive Informatics (ICICCI) (pp. 380-383).

Wu, J., Ping, L., Ge, X., Wang, Y., \& Fu, J. (2010). Cloud storage as the infrastructure of cloud computing. In 2010 International Conference on Intelligent Computing and Cognitive Informatics (ICICCI) (pp. 380-383). IEEE. doi:10.1109/ICICCI.2010.119

Yan, Q., \& Yu, F. R. (2015). Distributed denial of service attacks in software-defined networking with cloud computing. IEEE Communications Magazine, 53(4), 52-59. doi:10.1109/MCOM.2015.7081075

Yan, Q., \& Yu, F. R. (2015). Distributed denial of service attacks in software-defined networking with cloud computing. IEEE Communications Magazine, 53(4), 52-59. doi:10.1109/MCOM.2015.7081075

Jitendra Singh pursued $\mathrm{PhD}$ in computer science, in the area of cloud computing and completed the same in the year 2013. He has qualified the prestigious UGC-NET examination conducted by the UGC of India in year 2006. With over 15 years of experience in teaching and administration in higher education, and currently working with the college of University of Delhi. In addition, contributed as faculty member with Stratford University, USA, India Campus, as a part time faculty from more than 5 and half years. Beyond, he has contributed to dozens of research papers in the area of cloud computing, security. Several of them have been published in reputed journals indexed in Scopus, Inspec. In addition, he has also written books including 'Cloud computing for beginner to researcher', "Data structure simplified: Implementation with c++", "Python: Principles and practice". 